\title{
Generous Attitudes and Online Participation
}

\author{
FLOOR FIERS
}

AARON SHAW

Northwestern University, United States

\section{ESZTER HARGITTAI}

University of Zurich, Switzerland

\begin{abstract}
Some of the most popular websites depend on user-generated content produced and aggregated by unpaid volunteers. Contributing in such ways constitutes a type of generous behavior, as it costs time and energy while benefiting others. This study examines the relationship between contributions to a variety of online information resources and an experimental measure of generosity, the dictator game. Results suggest that contributors to any type of online content tend to donate more in the dictator game than those who do not contribute at all. When disaggregating by type of contribution, we find that those who write reviews, upload public videos, write or answer questions, and contribute to encyclopedic collections online are more generous in the dictator game than their non-contributing counterparts. These findings suggest that generous attitudes help to explain variation in contributions to review, question-and-answer, video, and encyclopedic websites.
\end{abstract}

Keywords: generosity, dictator game, online participation, user-generated content

Floor Fiers: floorfiers@u.northwestern.edu Date submitted: 2021-01-05

Copyright (C) 2021 (Floor Fiers, Aaron Shaw, Eszter Hargittai). Licensed under the Creative Commons Attribution-NonCommercial-NoDerivatives 4.0 International Public License. Available at: http://journalqd.org 
Over the last twenty years, the Internet has changed practices of sharing, enabling the rise of information goods created by large groups in online communities and websites (e.g., Benkler, 2006; Benkler et al., 2015; Kollock, 1999). In the case of publicly available information, Internet users draw to varying extents on user-generated content from learning about countless topics on one of the most popular websites, Wikipedia, to seeking answers on sites like Quora and Yahoo! Answers, and reading product reviews on retail websites (e.g., Band \& Gerafi, 2013; Gavilan et al., 2018). Much of this user-generated content is produced and aggregated by unpaid volunteers. Engaging in such behavior is arguably an act of generosity, since doing so costs the contributor some level of time and energy. Other users can benefit from these contributions by acquiring new knowledge that may help them form opinions and make decisions. In addition to providing resources for other users, the information goods that result from individual contributions hold immense value as public repositories of knowledge, bases for scientific research, and sources of innovations and profit (Band \& Gerafi, 2013; Geiger \& Halfaker, 2013; Hill \& Shaw, 2020; McMahon et al., 2017; Vincent et al., 2018).

Many scholars have attempted to explain what motivates individuals to spend so much time and energy advancing publicly shared information online, particularly as it concerns peer-production projects, which are organized in a distinctly decentralized and nonproprietary manner (for an overview, see Benkler et al., 2015). One explanation suggests that intrinsic prosocial attitudes fuel the decision to contribute to public goods (e.g., Algan et al., 2013). This study assesses this explanation by examining the relationship between contributing content online and generous behavior in the dictator game, a widely used measure of generosity (Bekkers, 2007; Eckel \& Grossman, 1996). By assessing the portion of a monetary reward that participants share with another study participant, the dictator game measures a person's willingness to lose some reward in the interest of sharing with an unknown other. Exploring whether certain online behaviors correlate with generosity as measured by the dictator game helps uncover whether and which types of generosity online may be linked to generosity in other facets of life. Few studies have 
examined this relationship. Exceptions tend to sample within one particular site or service, preventing them from comparing contributors to non-contributors and studying contribution behaviors across different domains of online content contribution (Algan et al., 2013; Ros-Galvez \& Rosa-Garcia, 2015). In contrast, this study uses a national sample to examine whether those who donate to the dictator game are also more generous in their contributions to various types of user-generated content online.

We use original survey data to compare generosity in dictator game donations among those who do and do not contribute information resources in several types of publicly available online content. We then present correlational analysis: Generosity in the dictator game is associated with contributions to any type of content as opposed to no contributions at all. When disaggregated by type of content, the findings suggest that generosity helps to explain variation in contributions to user-generated content on some types of websites, but not others. Specifically, donation behavior in the dictator game correlates with contributions to publicly available databases of reviews, questions and answers, videos, and encyclopedic content. However, the results do not demonstrate a similar relationship with contributions to online maps and citizen science projects. Further research would be needed to verify and explain these discrepancies. Logistic regression reveals that these results hold up when adjusting for a range of sociodemographic factors. The variation in results suggests that differentiating between specific contributions demonstrates that generosity in the dictator game only maps onto the generous behaviors of contributing content in some cases. The differentiated results suggest partial support for the idea that sites rely upon the generous attitudes of unpaid volunteers for content production. 


\section{Background}

\section{Generosity in the Digital Context}

Generosity and other kinds of selfless attitudes or behaviors have motivated decades of work across the social sciences, including a large literature that uses experimental games as an index of underlying generous attitudes (Fehr \& Fischbacher, 2003). Researchers have studied generous behaviors in the digital context through a variety of related concepts, including altruism, generosity, generalized exchange, and prosociality (e.g., Algan et al., 2013; Cheshire \& Antin, 2008; McAuliffe et al., 2018; Wright \& Li, 2011). While there are slight differences between the concepts, they are often used interchangeably, as they all refer to behaviors that come at a cost to the contributor and accrue benefits to others. In this paper, generosity refers to the act of voluntarily sharing or donating resources, whether monetary or otherwise, that benefit others.

Generosity and behaviors that embody this quality, such as contributing to free/libre and open-source software or making donations online, have been of interest to Internet researchers for decades (e.g., Benkler, 2006; Benkler et al., 2015; Cheshire \& Antin, 2008; Kollock, 1999). Broadly, Klisanin (2011) classified websites that facilitate generous behavior into three categories: (1) websites that rely upon "sharing of expertise created for the public good," (p. 3); (2) click-and-donate websites that benefit other people, animals or the environment; and (3) meta-cooperative endeavors to resolve large-scale social problems. While some of these categories overlap (e.g., educational projects in which users upload public videos or citizen science projects where user-generated information and data are shared for public consumption), this study primarily focuses on the first category of websites, which depend on user-generated content as the basis for publicly available information. While individual costs and benefits of contributing vary across content types, the sites and platforms hosting this information are intended to be useful for Internet users beyond the producers of the content. For example, while someone might experience social or psychological benefits from uploading a video to YouTube or posting a question to 
Quora, we consider these generous behaviors, as they ultimately contribute to a pool of information and resources that other Internet users can draw upon for their own benefit.

\section{Online Content Production}

Online content production has been widely studied in the field of digital inequality (for reviews, see Brake, 2014; Hargittai \& Jennrich, 2016). The practice of creating and sharing content is not distributed equally, meaning that divides exist between those who do and those who do not engage in such practices (e.g., Blank, 2013; Brake, 2014; Correa, 2010; Gan et al., 2018; Hargittai \& Walejko, 2008). For example, women (Hargittai \& Shaw, 2015; Schradie, 2015) and those who obtained lower levels of education (Hargittai $\&$ Walejko, 2008; Schradie, 2011) are less likely to share online than their counterparts. Similarly, prior research demonstrates that age (e.g., Haight et al., 2014; Hargittai \& Walejko, 2008), income (e.g., Haight et al., 2014) and urban/rural status (e.g., Haight et al., 2014; Schradie, 2011) explain varied online activity. Scholars have also emphasized the importance of considering Internet skills, as variation in skills can explain the engagement levels of various social groups (e.g., Hargittai \& Shaw, 2015; Hargittai \& Walejko, 2008).

While differences in background attributes and skill levels accounts for some variation in content contributions, there might be other factors explaining who produces more or less content online. This study adds to the literature by exploring the relationship between generosity and such behaviors. Contributing online is generous insofar that other Internet users can benefit from the resulting content in numerous ways. Such usergenerated content offers publicly available information resources to all Internet users. Thus, we examine whether an experimental measure of generosity correlates with these generous behaviors online. In line with previous work that has also differentiated among different types of online content sharing (Blank, 2013; Hargittai \& Walejko, 2008; Schradie, 2011), we examine six types of contributions to publicly available information online: posting reviews, asking or answering questions on question-and-answer sites, 
uploading public videos, contributing to encyclopedic collections, submitting edits to online maps, and contributing to citizen science projects.

Scholarship on the relationship between experimental measures of generosity and online engagement is scarce. Ros-Galvez and Rosa-Garcia (2015) use an adaptation of a dictator game to examine the level of generosity among users of two Spanish Internet forums. The study finds that individuals who are more active on the forums (i.e., number of posts) or have been active for longer (i.e., number of months since registration) tend to be more generous. Based on these findings, we might expect donations in the dictator game to correlate positively with content contributions. Other experimental evidence suggests, however, that the most active Wikipedia editors exhibit some prosocial attitudes including trust and reciprocity, but not generosity (Algan et al., 2013). We build on these studies in two main ways. First, we advance this research by taking into account contributions to a wider array of collective online goods, including reviews, online maps and citizen science projects. Little prior work has examined the relationship between generosity and any of these ways to contribute to publicly available content on the Internet. Second, we examine these relationships on a national sample. Previous studies on content production tend to sample based on the dependent variable, for example, by solely looking at Wikipedia editors (e.g., Algan et al., 2013; Collier \& Bear, 2012) or only recruiting through online forums (Ros-Galvez \& Rosa-Garcia, 2015). By analyzing a survey data set about a national sample of U.S. adults, we can further our understanding of those contributing to content online as compared to non-contributors.

\section{Methods}

This study relies upon survey data based on a national sample of 1,512 U.S. adults from the summer of 2016. The data set includes a comprehensive list of measures for online participation as well as an established measure for generosity (Bekkers, 2007). 
Data Collection

The independent research organization NORC at the University of Chicago administered the survey to their online AmeriSpeak panel. Consisting of U.S. adults, AmeriSpeak is a probability-based survey panel of the U.S. household population. The recruitment "includes additional coverage of hard-to-survey population segments such as rural and low-income households that are underrepresented in surveys relying on addressbased sampling" (NORC, n.d.).

After pretesting the survey with 23 respondents resulting in a few adjustments, the final survey ran from May 25 to July 5, 2016. Inclusion criteria included full completion of the survey and passing an attention check, which 1,512 people had done. The response rate was $37.8 \%$. The survey first asked about general Internet experiences and skills, followed by a battery of questions outside the scope of this paper (i.e., measuring prosocial behavior). Next, the survey inquired about content contribution behaviors examined here. The survey ended with the dictator game.

\section{Measures: Independent Variables}

Sociodemographic Factors. We use several background variables supplied by NORC on the survey respondents based on earlier data collection on their AmeriSpeak panel. We report age (in years) as a continuous variable. We coded gender as a dichotomous variable and education as a variable with three categories: high school or less, some college, and a Bachelor's degree or more. For descriptive and bivariate analyses, we coded race and ethnicity as six dummy variables: White, Hispanic, African American, Asian American, Native American, and Other. Due to sparse data on Native American respondents, we combined the Native American category with Other for the regression analysis. NORC provided income as an 18-category variable, which we recoded to the midpoints in order to make it a continuous variable and therefore, more easily interpretable. For the regression 
analysis, we use the square root of income, which resembles a normal distribution more closely. Lastly, we include a dichotomous variable for rural residence.

Generosity. To measure generosity, we use a survey-based adaptation of a unilateral dictator game (Bekkers, 2007). All participants were offered 2,000 points, which would then be converted to a payment of two U.S. dollars and subsequently issued as a bonus at the end of the study supplementing the pay respondents were already receiving for participation. The two-dollar incentive in the dictator game was in addition to the twodollar compensation respondents received for taking the survey. The survey questionnaire instructions explain, however, that they have the opportunity to share the points, and thus the payment, with another participant, to whom no points were offered. Subsequently, the survey asks the participant how many points they want to share. Every participant can share any number of points, ranging from no points at all $(0)$ to all of their points $(2,000)$.

After receiving the above instructions, but before being asked for their response, survey participants had to pass a question that tested their understanding of the payoffs. Only those who responded correctly saw the actual question through which they could access the bonus of 2,000 points. The number of bonus points that respondents decided to donate to a fellow respondent became our measure of their generosity. Of the 1,512 survey participants, 16 percent did not respond correctly to the test question and did not get the opportunity to receive and donate additional points. In Appendix A, we added results of ttests checking whether passing the comprehension test correlated with the various dependent variables. Among those who correctly answered the comprehension test and responded to the dictator game question, any points they shared were distributed to a different, randomly selected participant in the study. For all descriptive and bivariate analyses, we use the raw number of points donated (0-2000). We convert the points donated to z-scores for the regression analysis. 


\section{Measures: Dependent Variables}

In the survey, we asked respondents: "have you ever done any of the following?" and provided a list of different online activities. Respondents reported either having done each activity in the past or not (the answer options were "yes" and "no"). We classified these activities into six types, which we describe below. Appendix B contains the exact wording for all survey items about online engagement.

Posting Reviews. The question included three activities concerning posting reviews: "posted a review on Amazon," "posted a review on TripAdvisor," and "posted a review on Yelp." We aggregated these three into one dichotomous variable, indicating whether the respondent has ever posted a review on at least one of the three sites.

Asking or Answering Questions on Q\&A Sites. We inquired whether respondents had ever "asked or answered a question in an online forum such as on Facebook or Twitter" or "in a social Q\&A site (like Quora, Yahoo! Answers, or StackOverflow)". We combined these two variables into one measure.

Uploading Public Videos. The activities included having "posted a video publicly (like on YouTube or Facebook)," which is a dichotomous measure on its own.

Contributing to Encyclopedic Collections. The list of activities asked about ever having "contributed information to IMDB." Later in the survey, we asked: "Have you ever edited a Wikipedia page by fixing a mistake or adding new material?" We combined these two variables into one dichotomous measure of contributing to encyclopedic collections on the Internet.

Submitting Edits to Online Maps. The list of activities also asked about having "submitted edits to an online map (like OpenStreet Map or Google Map Maker)," which is also a dichotomous measure on its own. 
Contributing to Online Citizen Science Projects. The list of activities included ever having "contributed to a citizen science project online (like Zooniverse or Foldit)," which is a binary zero versus one measure on its own.

Contributing to Any of the Sites. Finally, we combined all six types of activities into an aggregate measure of producing and sharing publicly available content. This binary variable indicates whether respondents contributed or not to online content in any of the ways described above.

\section{Sample}

Table 1 contains descriptive statistics about the sample. The ages of respondents range from 18 to 94 , with a mean age of 48.7 years. A little over half, 51\%, are female. The majority at $71 \%$ are White, $12 \%$ are Hispanic, 11\% African American, 3\% Asian American, with 3\% reporting Other. Just over a quarter (26\%) had obtained a high school degree or less, $32 \%$ had completed some college, and $43 \%$ at least a Bachelor's degree. The mean income among respondents is \$71,478 USD (median: 54,999 USD), with $62 \%$ of the sample being employed. The majority (61\%) lived with a partner with an average of 2.6 people per household. A small portion (13\%) of respondents lived in rural areas.

\section{Analysis}

We first calculate descriptive statistics for both the independent and dependent variables. We then compare dictator game donations for those who contributed content (overall as well as by type of activity) and those who did not. Finally, we fit logistic regressions for each of the dependent variables in order to examine how dictator game donations relate to the outcome activities when controlling for age, gender, income, education, rural/urban status, and race and ethnicity. 


\section{Results}

\section{Descriptive Analysis}

Table 1. Descriptive Statistics

\begin{tabular}{lcccc}
\hline & Percent & Mean & SD & N \\
\hline Background & & & & \\
Age (18 - 94) & & 48.74 & 16.87 & 1512 \\
Income in U.S. \$1,000s (2.5 - 225) & & 71.48 & 54.4 & 1512 \\
Household size (1 - 6) & & 2.61 & 1.32 & 1512 \\
Female & 51 & & & 1512 \\
Coupled & 61 & & & 1512 \\
Employed & 62 & & 1512 \\
Rural resident & 13 & & 1512 \\
Education & & & \\
High school or less & 26 & & 1512 \\
Some college & 32 & & 1512 \\
Bachelor's or higher & 43 & & 1512 \\
Race \& Ethnicity & & & 1511 \\
White & 71 & & 1511 \\
Hispanic & 12 & & 1511 \\
Black & 11 & & 1511 \\
Asian & 3 & & 1511 \\
Other & 3 & & \\
\hline
\end{tabular}

Note. For interval measures the range (min. - max.) appears in parentheses.

The number of donated points ranged between 0 and $2000(\mu=749, \sigma=479)$. This indicates that the average respondent donated $37.5 \%$ of their bonus, which is slightly higher than the average percentage donated in other studies using some form of a dictator game (for a meta-analysis, see Engel, 2011). Figure 1 provides the variable's distribution. Table 2 contains the descriptive statistics for the dependent variables. The majority (79\%) of 
respondents indicated that they have contributed at least one of the types of content. About half of the respondents had engaged in each of the following two activities: posted reviews and asked or answered questions on Q\&A forums. Over a third (39\%) had ever uploaded a public video. Even fewer contributed to either Wikipedia or IMDB at $9 \%$, only $7 \%$ submitted edits to online maps, and only $2 \%$ reported contributing to citizen science projects.

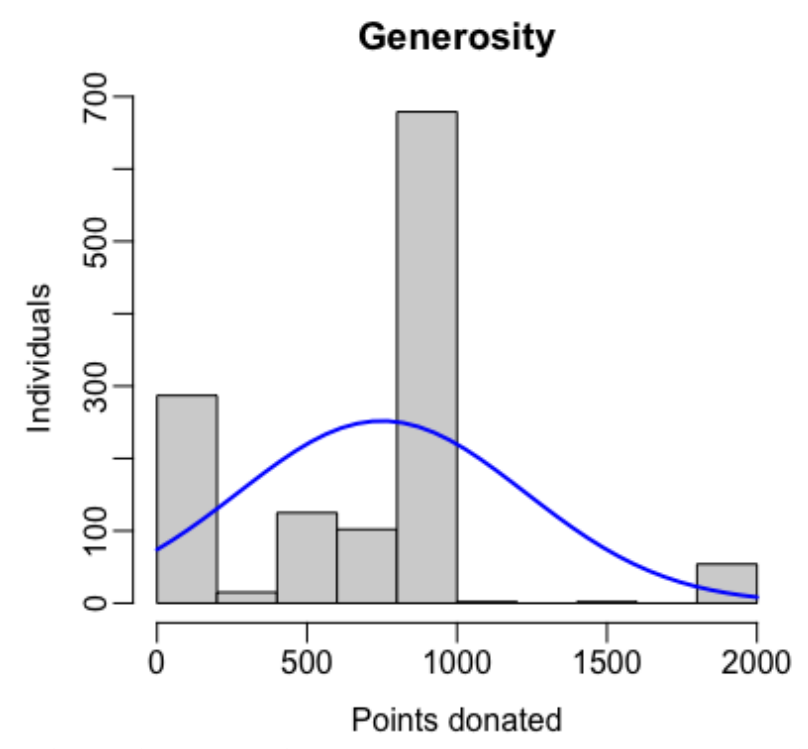

Figure 1. Distribution of generosity as measured by donated points in the dictator game.

\section{Bivariate Analysis}

Table 3 provides the results of the bivariate analyses of points donated in the dictator game over each outcome for all the dependent variables. In every comparison, the observed difference of means is greater than zero (i.e., those who engage in the type of online contribution in question donate more in the dictator game, on average, than those who do not contribute online). For four out of six types of user-generated content, a t-test rejects the null hypothesis of no difference at the conventional level of $\mathrm{p}<0.05$, suggesting strong evidence that the mean of donated points is higher for those who contribute to user- 
generated content than the mean of those who did not. Specifically, those who have at least once posted a review donate on average 776 points, whereas their counterparts donate on average 719 points. Contributors to a Q\&A site, whether by asking or by answering questions, have donated an average of 786 points, whereas those who have not contributed to these sites gave away an average of 711 points. Those who have ever uploaded a public video donated an average of 798 points, as compared to an average of 719 points donated by those who have never uploaded a public video. Lastly, contributors to Wikipedia or IMDB donated more points than their counterparts, with the former donating an average of 870 points and the latter 736 points. While we cannot reject the null hypothesis in the means of donated points by contributors to online maps and citizen science projects versus noncontributors, the difference in means is large as well.

Table 2. Descriptive Statistics for Dependent Variables

\begin{tabular}{lcc}
\hline & Percent & $\mathrm{N}$ \\
\hline Contribute to any of the sites & 79 & 1512 \\
Posting reviews & 53 & 1509 \\
Asking or answering questions & 50 & 1511 \\
Uploading public videos & 39 & 1508 \\
Contributing to encyclopedic collections & 9 & 1509 \\
Submitting edits to online maps & 7 & 1507 \\
Contributing to citizen science projects & 2 & 1509 \\
\hline
\end{tabular}

For the aggregate measure concerning contribution of any type of content, a t-test rejects the null hypothesis of no difference, suggesting that the mean of donated points is higher for those who contributed to any of the six types of online resources, as compared to those who contributed to none.

\section{Regression}

Table 4 presents the logistic regression results predicting contributions to publicly available information resources, both for all six individual types of activities and for the 
measure indicating contribution in any of the activities. The coefficients are raw log-odds and we include standard errors in parentheses. The regression results show that the findings of the bivariate analyses generally hold when controlling for various sociodemographic factors. The dictator game correlates with four out of six contribution activities: posting reviews, uploading public videos, asking or answering questions on Q\&A forums, and editing Wikipedia or IMDB. The regression analysis also indicates that dictator game donations correlate with the aggregate measure of contributing to any type of content versus none. In some cases, the regression coefficients are smaller than the coefficients of various sociodemographic factors, such as education and gender.

Table 3. Bivariate Summary. Conditional Means of Donated Points by Online Content Contribution.

\begin{tabular}{|c|c|c|c|c|}
\hline & $\begin{array}{l}\text { Mean donated } \\
\text { points of } \\
\text { contributors } \\
\end{array}$ & $\begin{array}{c}\text { Mean } \\
\text { donated } \\
\text { points of non- } \\
\text { contributors }\end{array}$ & $\begin{array}{l}\text { Difference in means } \\
{[95 \% \mathrm{CI}]}\end{array}$ & $\mathrm{p}$-value \\
\hline $\begin{array}{l}\text { Contributing to any of the } \\
\text { sites }\end{array}$ & 764 & 698 & $\begin{array}{c}66.41 \\
{[1.05,131.77]}\end{array}$ & 0.05 \\
\hline Posting reviews & 776 & 719 & $\begin{array}{c}56.78 \\
{[3.39,110.18]}\end{array}$ & 0.04 \\
\hline $\begin{array}{l}\text { Asking or answering } \\
\text { questions }\end{array}$ & 796 & 719 & $\begin{array}{c}76.77 \\
{[23.07,130.46]}\end{array}$ & 0.01 \\
\hline Uploading public videos & 786 & 711 & $\begin{array}{c}74.67 \\
{[21.91,127.43]}\end{array}$ & 0.01 \\
\hline $\begin{array}{l}\text { Contributing to } \\
\text { encyclopedic collections }\end{array}$ & 870 & 736 & $\begin{array}{c}133.77 \\
{[49.56,217.97]}\end{array}$ & 0.00 \\
\hline $\begin{array}{l}\text { Submitting edits to online } \\
\text { maps }\end{array}$ & 799 & 746 & $\begin{array}{c}53.56 \\
{[-61.61,168.73]}\end{array}$ & 0.36 \\
\hline $\begin{array}{l}\text { Contributing to citizen } \\
\text { science projects }\end{array}$ & 808 & 748 & $\begin{array}{c}59.72 \\
{[-186.78,306.22]}\end{array}$ & 0.62 \\
\hline
\end{tabular}

Note: $\mathrm{p}=<0.05$ are in bold. 
Table 4. Logistic Regression Model Results Predicting Online Content Contribution.

\begin{tabular}{|c|c|c|c|c|c|c|c|}
\hline & Any & Review & Q\&A & Video & \multicolumn{2}{|c|}{$\begin{array}{l}\text { Encyclopedir Online maps } \\
\text { collection }\end{array}$} & \multirow{2}{*}{ 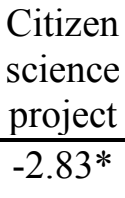 } \\
\hline \multirow[t]{2}{*}{ (Intercept) } & $2.46^{* *}$ & -0.16 & $1.00 * *$ & $1.60 * *$ & -0.48 & $-1.99 * *$ & \\
\hline & $(-0.34)$ & $(-0.26)$ & $(-0.27)$ & $(-0.28)$ & $(-0.43)$ & $(-0.51)$ & $(-1.2)$ \\
\hline \multirow[t]{2}{*}{ Donated points } & $0.14^{*}$ & $0.12 *$ & $0.16^{* *}$ & $0.17 * *$ & $0.24 *$ & 0.12 & 0.03 \\
\hline & $(-0.07)$ & $(-0.06)$ & $(-0.06)$ & $(-0.06)$ & $(-0.1)$ & $(-0.12)$ & $(-0.2)$ \\
\hline \multirow[t]{2}{*}{ Age } & $-0.04 * *$ & $-0.02 * *$ & $-0.03 * *$ & $-0.04 * *$ & $-0.04 * *$ & $-0.02 *$ & $-0.05 * *$ \\
\hline & $(-0.005)$ & $(-0.004)$ & $(-0.004)$ & $(-0.004)$ & $(-0.01)$ & $(-0.01)$ & $(-0.01)$ \\
\hline \multirow[t]{2}{*}{ Female } & 0.14 & 0.12 & $0.36^{* *}$ & 0.02 & $-0.71 * *$ & $-0.62 *$ & 0.01 \\
\hline & $(-0.14)$ & $(-0.12)$ & $(-0.12)$ & $(-0.12)$ & $(-0.2)$ & $(-0.25)$ & $(-0.41)$ \\
\hline \multirow[t]{2}{*}{ Rural resident } & 0.13 & -0.15 & 0.22 & 0.11 & 0.2 & 0.09 & 0.45 \\
\hline & $(-0.21)$ & $(-0.17)$ & $(-0.18)$ & $(-0.19)$ & $(-0.29)$ & $(-0.36)$ & $(-0.59)$ \\
\hline \multirow[t]{2}{*}{ Income } & 0.01 & $0.08^{* *}$ & -0.004 & -0.02 & -0.03 & 0.01 & $-0.16^{*}$ \\
\hline & $(-0.02)$ & $(-0.02)$ & $(-0.02)$ & $(-0.02)$ & $(-0.03)$ & $(-0.04)$ & $(-0.07)$ \\
\hline \multicolumn{8}{|c|}{ Education (base $=$ HS or less) } \\
\hline \multirow[t]{2}{*}{ Some college } & $0.68^{* *}$ & $0.83 * *$ & $0.54 * *$ & 0.24 & $0.57^{\dagger}$ & 0.25 & 1.43 \\
\hline & $(-0.19)$ & $(-0.16)$ & $(-0.16)$ & $(-0.17)$ & $(-0.32)$ & $(-0.33)$ & $(-1.11)$ \\
\hline BA or higher & $0.75^{* *}$ & $0.79 * *$ & $0.28^{\dagger}$ & 0.03 & $1.17 * *$ & 0.05 & $2.92 * *$ \\
\hline
\end{tabular}


Fiers et al.

Journal of Quantitative Description: Digital Media 1(2021) 16

$$
\begin{array}{llllll}
(-0.18) & (-0.16) \quad(-0.16) \quad(-0.17) \quad(-0.3) \quad(-0.33) & (-1.05)
\end{array}
$$

Race \& ethnicity (base $=$ White)

\begin{tabular}{lccccccc} 
Hispanic & $-0.39^{\dagger}$ & $-0.41^{*}$ & $-0.34^{\dagger}$ & 0.04 & -0.24 & 0.28 & -0.83 \\
\multirow{3}{*}{ Black } & $(-0.23)$ & $(-0.19)$ & $(-0.19)$ & $(-0.19)$ & $(-0.31)$ & $(-0.36)$ & $(-0.77)$ \\
& 0.36 & 0.08 & 0.18 & 0.26 & -0.16 & $0.78^{*}$ & -1.46 \\
\multirow{2}{*}{ Asian } & $(-0.27)$ & $(-0.2)$ & $(-0.2)$ & $(-0.2)$ & $(-0.33)$ & $(-0.33)$ & $(-1.04)$ \\
& $-1.02^{* *}$ & -0.06 & $-0.79^{*}$ & -0.57 & $-1.30^{\dagger}$ & -0.21 & 0.28 \\
\multirow{2}{*}{ Other } & $(-0.36)$ & $(-0.34)$ & $(-0.35)$ & $(-0.36)$ & $(-0.75)$ & $(-0.75)$ & $(-0.78)$ \\
& 0.41 & 0.23 & $0.80^{\dagger}$ & 0.5 & 0.09 & 0.19 & 0.77 \\
& $(-0.51)$ & $(-0.39)$ & $(-0.42)$ & $(-0.4)$ & $(-0.64)$ & $(-0.76)$ & $(-1.11)$ \\
\hline Observations & 1,265 & 1,262 & 1,262 & 1,264 & 1,262 & 1,260 & 1,262 \\
Log Likelihood & -626.88 & -823.52 & -765.99 & -822.73 & -370.24 & -282.57 & -107.36 \\
AIC & $1,277.76$ & $1,671.04$ & $1,555.98$ & $1,669.45$ & 764.48 & 589.14 & 238.72 \\
\hline
\end{tabular}

Note: For regression coefficients, ${ }^{\dagger} \mathrm{p}<0.1 ;{ }^{* *} \mathrm{p}<0.05 ; * * * \mathrm{p}<0.01$. 


\section{Discussion}

In this study, we find evidence that those producing any type of publicly available content tend to donate more points in a unilateral dictator game than those who do not produce such content. When looking at various content contribution types separately, we find that donating in the dictator game correlates with content contribution for four out of six types considered in this study. Specifically, those who write reviews, write or answer questions, upload public videos, and contribute to Wikipedia or IMDB are more generous in the dictator game than their counterparts. The results hold in bivariate analysis of differences-in-means as well as multiple logistic regressions adjusting for participants' age, gender, income, education, urban/rural status, and race/ethnicity. This suggests that people with more generous behavior in the dictator game also tend to engage in some more generous behaviors online. For the other two contribution activities, contributing to editing online maps and citizen science projects, we cannot reject the null-hypothesis of no difference in dictator game generosity using either a t-test or the multiple regression. However, the observed difference of means in dictator game donations and the point estimate for the regression parameter on dictator game donations are greater than zero. Notably, even with the activities where we find the dictator game correlates with online content contributions, we also find that some sociodemographic factors are stronger predictors of those content contributions than dictator game donations.

The results extend prior literature on online content creation by suggesting that generous behavior in the dictator game correlates with some types of contributions to usergenerated content, but not others. In addition to sociodemographic factors examined in earlier studies, generous attitudes help to explain variation in several types of online content contributions. The findings are in agreement with prior research on contributions to online forums (Ros-Galvez \& Rosa-Garcia, 2015). In contrast, our results concerning encyclopedic databases deviate from the results of Algan et al. (2013), who do not find a correlation between the dictator game and Wikipedia editing. However, both studies support the role of prosocial attitudes in explaining variation in Wikipedia editing. We do 
not find evidence of a similar relationship between donations in the dictator game and all types of content contributions. Future research could assess why these discrepancies emerge, for example by focusing on the different nature of contribution activities, as well as explore whether any discrepancies might be used to inform future site designs.

The variation between the types of content contribution reinforces the conclusions drawn in prior studies that content production should not be treated as homogenous activity (e.g., Hargittai \& Walejko, 2008; Schradie, 2011). Research on content creation and online activity more broadly should consider various ways to contribute content and behave generously. It is noteworthy, however, that the size of contributor versus non-contributor groups varied strongly depending on the contribution type. For the two contribution types for which our analyses yield null findings, the number of contributors is particularly low: $7 \%$ of the respondents $(n=102)$ had edited online maps before and only $2 \%(n=31)$ have contributed to citizen science projects. Future research could analyze larger samples to further explore these relationships.

Another limitation is the set-up of the survey that requires respondents to pass the comprehension test before being able to participate in the dictator game. In two out of six content contribution types, passing the comprehension correlates with content contribution (see Appendix A for t-test results). This implies that the correlations between generosity measured in the dictator game and the online contribution behaviors may be due to an unobserved association between some underlying factor that determines comprehension of the instrument rather than generosity per se. We are unable to evaluate this possibility directly as the comprehension test ensures the validity of the dictator game measure. Alternative measurement strategies would be necessary to overcome this concern.

Future research can also improve the measures of content contribution. One way to do so would be by including more types of contribution activities and further specifying the activities. In this study, the level of specificity is not consistent across all measures, where some survey questions asked about contributions to a specific website, while others 
inquired about a more general behavior. Similarly, of the questions inquiring about a general behavior, only some of the questions provided an example of a website. The descriptions of the activities in the survey can also be more specific, e.g. distinguishing between asking versus answering a question on a social Q\&A site. Particularly when examining content contribution as a generous behavior, variables measuring intentions behind content contribution can provide another level of understanding. This might clarify the role of generous attitudes in motivating such behaviors. Alternatively, future research can substitute the survey questions regarding online behavior with unobtrusively collected trace data to overcome issues of potential bias with self-reported data, the methods of this study. Prior research has demonstrated the potential lack of accuracy in self-report measures of Internet use (e.g., Araujo et al., 2017; Scharkow, 2016), so we treat the measures of content contribution with care. We are unable to estimate this directly using our data Nonetheless, we expect the risk of overreporting to be small in this study, since all content contribution measures are binary (i.e., either a respondent has performed a certain activity or not). In contrast, the lack of accuracy reported by Araujo and colleagues (2017) as well as by Scharkow (2016) concerns self-report estimates of the amount one engages with the Internet.

\section{Conclusion}

This study examined the relationship between generosity in the dictator game and the act of contributing to publicly available user-generated content online, an activity that comes at a cost for the contributor and accrues benefits for others. We find evidence that those contributing to any type of content online donate more points in the dictator game than those who do not contribute at all. Disaggregating the various types of content contribution demonstrates that this relationship holds for four out of six types of contributions: writing reviews, posing or answering questions on forums, uploading public videos, and contributing to encyclopedic databases like Wikipedia and IMDB. While the observed differences in means are positive for the remaining contribution types as well, we 
find no evidence of a relationship between donated points in the dictator game and two activities: contributing to editing online maps and citizen science projects.

The findings suggest that generosity helps to explain variation in content contribution in most cases, which supports the idea that generous and pro-social attitudes fuels the decision to contribute online. However, generosity does not explain variation in all types of content contribution. Regardless of a definitive explanation for the variation in results, differentiating between types of content contributions proved valuable for understanding the relationship between the dictator game and the online content contributions. Since treating content contribution as a homogenous activity might have led to incomplete conclusions, future work on the topic should continue to make distinctions between different types of contributions.

These findings also indicate that generosity only forms part of the basis upon which Internet users decide to spend time, energy, and knowledge on the production and aggregation of online content. Some sites reliant on user-generated content might count on their users' generous attitudes, while others might need to appeal to other traits and attitudes. Overall, generosity provides an important piece to understanding the origins of user-generated content and its impact for the individual Internet users, researchers, and companies who draw on such content for various purposes.

\section{Acknowledgements}

Staff at NORC at the University of Chicago administered the survey discussed in this study. The authors are grateful to Merck (Merck is known as MSD outside the United States and Canada) as well as the Robert and Kaye Hiatt Fund at Northwestern University for support. 
Journal of Quantitative Description: Digital Media 1(2021) Generosity and Online Participation 21

\section{References}

Algan, Y., Benkler, Y., Fuster Morell, M., \& Hergueux, J. (2013). Cooperation in a peer production economy: Experimental evidence from Wikipedia. [Working Paper]. http://dx.doi.org/10.2139/ssrn.2843518

Araujo, T., Wonneberger, A., Neijens, P., \& Vreese, C. de. (2017). How Much Time Do You Spend Online? Understanding and Improving the Accuracy of Self-Reported Measures of Internet Use. Communication Methods and Measures, 11(3), 173190. https://doi.org/10.1080/19312458.2017.1317337

Band, J., \& Gerafi, J. (2013). Wikipedia's Economic Value [SSRN Scholarly Paper]. Social Science Research Network. http://dx.doi.org/10.2139/ssrn.2338563

Bekkers, R. (2007). Measuring Altruistic Behavior in Surveys: The All-or-Nothing Dictator Game. Survey Research Methods, 1(3), 139-144. https://doi.org/10.18148/srm/2007.v1i3.54

Benkler, Y. (2006). The Wealth of Networks: How Social Production Transforms Markets and Freedom. Yale University Press.

Benkler, Y., Shaw, A., \& Hill, B. M. (2015). Peer production: A form of collective intelligence. In T. W. Malone \& M. S. Bernstein (Eds.), Handbook of Collective Intelligence (pp. 175-204). MIT Press.

Blank, G. (2013). Who creates content? Information, Communication \& Society, 16(4), 590-612. https://doi.org/10.1080/1369118X.2013.777758

Brake, D. R. (2014). Are we all online content creators now? Web 2.0 and digital divides. Journal of Computer-Mediated Communication, 19(3), 591-609. https://doi.org/10.1111/jcc4.12042

Cheshire, C., \& Antin, J. (2008). The social psychological effects of feedback on the production of Internet information pools. Journal of Computer-Mediated Communication, 13(3), 705-727. https://doi.org/10.1111/j.10836101.2008.00416.x

Collier, B., \& Bear, J. (2012). Conflict, criticism, or confidence: An empirical examination of the gender gap in Wikipedia contributions. Proceedings of the 
ACM 2012 Conference on Computer Supported Cooperative Work, 383-392. https://doi.org/10.1145/2145204.2145265

Correa, T. (2010). The participation divide among "online experts": Experience, skills and psychological factors as predictors of college students' web content creation. Journal of Computer-Mediated Communication, 16, 71-92. https://doi.org/10.1111/j.1083-6101.2010.01532.x

Eckel, C. C., \& Grossman, P. J. (1996). Altruism in anonymous dictator games. Games and Economic Behavior, 16(2), 181-191. https://doi.org/10.1006/game.1996.0081

Engel, C. (2011). Dictator games: A meta study. Experimental Economics, 14(4), 583610. https://doi.org/10.1007/s10683-011-9283-7

Fehr, E., \& Fischbacher, U. (2003). The nature of human altruism. Nature, 425(6960), 785-791. https://doi.org/10.1038/nature02043

Gan, E. F., Hill, B. M., \& Dasgupta, S. (2018). Gender, feedback, and learners' decisions to share their creative computing projects. Proceedings of the ACM on HumanComputer Interaction, 2(CSCW), 54:1-54:23. https://doi.org/10.1145/3274323

Gavilan, D., Avello, M., \& Martinez-Navarro, G. (2018). The influence of online ratings and reviews on hotel booking consideration. Tourism Management, 66, 53-61. https://doi.org/10.1016/j.tourman.2017.10.018

Geiger, R. S., \& Halfaker, A. (2013). When the levee breaks: Without bots, what happens to Wikipedia's quality control processes? Proceedings of the 9th International Symposium on Open Collaboration (OpenSym '13), 6:1-6:6. https://doi.org/10.1145/2491055.2491061

Haight, M., Quan-Haase, A., \& Corbett, B. A. (2014). Revisiting the digital divide in Canada: The impact of demographic factors on access to the internet, level of online activity, and social networking site usage. Information, Communication \& Society, 17(4), 503-519. https://doi.org/10.1080/1369118X.2014.891633

Hargittai, E., \& Jennrich, K. (2016). The online participation divide. In L. A. Friedland \& M. Lloyd (Eds.), The communication crisis in America, and how to fix it (pp. 199213). Palgrave Macmillan. https://doi.org/10.1057/978-1-349-94925-0_13

Hargittai, E., \& Shaw, A. (2015). Mind the skills gap: The role of internet know-how and 
Journal of Quantitative Description: Digital Media 1(2021) Generosity and Online Participation 23

gender in differentiated contributions to Wikipedia. Information, Communication \& Society, 18(4), 424-442. https://doi.org/10.1080/1369118X.2014.957711

Hargittai, E., \& Walejko, G. (2008). The participation divide: Content creation and sharing in the digital age. Information, Communication \& Society, 11(2), 239256. https://doi.org/10.1080/13691180801946150

Hill, B. M., \& Shaw, A. D. (2020). The most important laboratory for social scientific and computing research in history. In J. M. Jr. Reagle \& J. L. Koerner (Eds.), Wikipedia@20: Stories of an incomplete revolution. MIT Press. https://library.oapen.org/handle/20.500.12657/43467

Klisanin, D. (2011). Is the Internet giving rise to new forms of altruism. Media Psychology Review [Online], 3(1), 1-11.

Kollock, P. (1999). The economies of online cooperation: Gifts and public goods in cyberspace. In M. Smith \& P. Kollock (Eds.), Communities in cyberspace (pp. 220-239). Routledge.

McAuliffe, W. H. B., Forster, D. E., Philippe, J., \& McCullough, M. E. (2018). Digital altruists: Resolving key questions about the empathy-altruism hypothesis in an Internet sample. Emotion, 18(4), 493-506. https://doi.org/10.1037/emo0000375

McMahon, C., Johnson, I. L., \& Hecht, B. J. (2017). The substantial interdependence of Wikipedia and Google: A case study on the relationship between peer production communities and information technologies. International AAAI Conference on Web and Social Media (ICWSM 2017), 142-151. https://ojs.aaai.org/index.php/ICWSM/article/view/14883

NORC. (n.d.). Amerispeak: NORC's breakthrough panel-based research platform. https://perma.cc/2TBM-KLUJ. Retrieved February 5, 2018, from http://www.norc.org/Research/Capabilities/pages/amerispeak.aspx

Ros-Galvez, A., \& Rosa-Garcia, A. (2015). Private provision of a public good: Cooperation and altruism of internet forum users. International Journal of the Commons, 9(2), 720-743. https://doi.org/10.18352/ijc.554

Scharkow, M. (2016). The Accuracy of Self-Reported Internet Use-A Validation Study Using Client Log Data. Communication Methods and Measures, 10(1), 13-27. 
https://doi.org/10.1080/19312458.2015.1118446

Schradie, J. (2011). The digital production gap: The digital divide and Web 2.0 collide. Poetics, 39(2), 145-168. https://doi.org/16/j.poetic.2011.02.003

Schradie, J. (2015). The gendered digital production gap: Inequalities of affluence. In L. Robinson, S. R. Cotten, \& J. Schulz (Eds.), Communication and information technologies annual (Vol. 9, pp. 185-213). Emerald Group Publishing Limited. http://www.emeraldinsight.com/doi/full/10.1108/S2050-206020150000009008

Vincent, N., Johnson, I., \& Hecht, B. (2018). Examining Wikipedia with a broader lens: Quantifying the value of Wikipedia's relationships with other large-scale online communities. Proceedings of the 2018 CHI Conference on Human Factors in Computing Systems, 566:1-566:13. https://doi.org/10.1145/3173574.3174140

Wright, M. F., \& Li, Y. (2011). The associations between young adults' face-to-face prosocial behaviors and their online prosocial behaviors. Computers in Human Behavior, 27(5), 1959-1962. https://doi.org/10.1016/j.chb.2011.04.019 